\title{
A GENERALIZED PROJECTION DECOMPOSITION IN ORLICZ-BOCHNER SPACES
}

\author{
HENRYK HUDZIK \\ Faculty of Mathematics and Computer Science, Adam Mickiewicz University \\ Umultowska 87, 61-614 Poznań, Poland \\ E-mail: hudzik@amu.edu.pl \\ RYSZARD PEUCIENNIK \\ Institute of Mathematics, Poznań University of Technology \\ Piotrowo 3A, 60-965 Poznań, Poland \\ E-mail: rplucien@math.put.poznan.pl \\ YUWEN WANG \\ School of Mathematics and Computer Science, Harbin Normal University \\ Harbin 150080, P.R. China \\ E-mail:wangyuwen2003@sohu.com
}

\begin{abstract}
In this paper, a precise projection decomposition in reflexive, smooth and strictly convex Orlicz-Bochner spaces is given by the representation of the duality mapping. As an application, a representation of the metric projection operator on a closed hyperplane is presented.
\end{abstract}

1. Introduction. It is well known that if $K$ is a closed convex cone (resp. closed linear subspace) in a Hilbert function space, we have the Moreau (resp. Riesz) decomposition theorem $x=P_{K}(x)+P_{K^{0}}(x)$ (resp. $x=P_{K}(x)+P_{K^{\perp}}(x)$ ), but the decomposition does not hold in arbitrary Banach function spaces. Many authors have attempted to generalize it. In 1995, Y. W. Wang and Z. W. Li [15] (resp. in 2001, Y. W. Wang and H. Wang [16]) obtained a decomposition by using the metric projection operator (i.e. projector $\pi_{L}$ )

$$
x=\pi_{L}(x)+x_{2}, \forall x \in X,
$$

2000 Mathematics Subject Classification: 46B20, 46E30.

Key words and phrases: Orlicz-Bochner space, projection decomposition, duality mapping, hyperplane.

Research of Y. Wang was supported by the National Science Foundation Grant (10471023) of China.

The paper is in final form and no version of it will be published elsewhere. 
where $L$ is a closed convex cone (resp. a Chebyshev subspace) of a real reflexive strictly convex Banach space (resp. a general Banach space) $X, x_{2} \in J^{-1} L^{\perp}$ and $x_{2}$ is not definite. In 1998, Ya. I. Alber [1] obtained another decomposition in a reflexive strictly convex smooth Banach space $X$ :

$$
x=J^{-1} \Pi_{K^{0}} J x+w,
$$

where $K$ is a closed convex cone in $X, J: X \rightarrow X^{*}$ is the duality mapping of $X, w \in K$ and $w$ is not definite, so their decompositions are semi-definite. W. Song and Z. J. Cao [14] investigated this problem in a more precise and general form. The aim of this paper is to give a precise representation of such a decomposition in Orlicz-Bochner spaces $L_{\Phi}(X)$.

2. Definitions and preliminary lemmas. We denote by $(G, \Sigma, \mu)$ a measure space in the $n$-dimensional Euclidean space $\mathbb{R}^{n}$ with $0<\mu G<\infty$, by $\mathbb{R}$ the set of real numbers, by $\left(X,\|\cdot\|_{X}\right)$ a reflexive real Banach space, by $\left(X^{*},\|\cdot\|_{X^{*}}\right)$ the dual space of $X$, by $\left\langle x^{*}, x\right\rangle$ the dual pairing of $x^{*} \in X^{*}$ and $x \in X$ and by $L^{0}(G, X)$ the linear space of all $\mu$-equivalent classes of strongly measurable functions $x: G \rightarrow X$.

A convex and even function $\Phi: \mathbb{R} \rightarrow \mathbb{R}_{+}$is called an Orlicz function if $\Phi(0)=0$, $\Phi(u)>0$ for $u \neq 0$, and

$$
\lim _{u \rightarrow 0} \frac{\Phi(u)}{|u|}=0, \quad \lim _{u \rightarrow \infty} \frac{\Phi(u)}{|u|}=\infty .
$$

For any Orlicz function $\Phi$, we define its complementary function $\Psi: \mathbb{R} \rightarrow \mathbb{R}_{+}$by the formula

$$
\Psi(v)=\sup _{u>0}\{u|v|-\Phi(u)\}
$$

for every $v \in \mathbb{R}$. The function $\Psi$ is also an Orlicz function (see [8], [4]).

We say that an Orlicz function $\Phi$ satisfies the $\Delta_{2}$-condition (write $\Phi \in \Delta_{2}$ ) if there exist constants $K>1$ and $u_{0}>0$ such that

$$
\Phi(2 u) \leq K \Phi(u) \text { for any } u \geq u_{0} .
$$

We say that an Orlicz function $\Phi$ satisfies the $\nabla_{2}$-condition (write $\Phi \in \nabla_{2}$ ) if its complementary function $\Psi$ satisfies the $\Delta_{2}$-condition.

Denote by small letters $\varphi$ and $\psi$ the right hand side derivatives of the Orlicz functions $\Phi$ and $\Psi$, respectively.

The space

$$
L_{\Phi}(X)=\left\{x \in L^{0}(G, X): \exists k>0 \text { s.t. } \rho_{\Phi}(k x)=\int_{G} \Phi\left(k\|x(t)\|_{X}\right) d t<\infty\right\}
$$

equipped with the so called Orlicz norm

$$
\|x\|_{\Phi}^{0}=\sup \left\{\left|\int_{G}\langle y(t), x(t)\rangle d t\right|: y \in L_{\Psi}\left(X^{*}\right), \rho_{\Psi}(y) \leq 1\right\}
$$

or with the Luxemburg norm

$$
\|x\|_{\Phi}=\inf \left\{k>0: \rho_{\Phi}\left(\frac{x}{k}\right) \leq 1\right\}
$$


is said to be an Orlicz-Bochner space (see [7]). In the following $L_{\Phi}(X)\left(\right.$ resp. $L_{\Phi}^{0}(X)$ ) denotes the Orlicz-Bochner space equipped with the Luxemburg norm (resp. equipped with the Orlicz norm). If $X=\mathbb{R}$, the Orlicz-Bochner spaces become the classical Orlicz spaces (see [10] or [17]) and they are denoted by $L_{\Phi}$ and $L_{\Phi}^{0}$, respectively.

The following Hölder inequalities

$$
\begin{aligned}
& \left|\int_{G}\langle y(t), x(t)\rangle d t\right| \leq\|x\|_{\Phi}\|y\|_{\Psi}^{0}, \\
& \left|\int_{G}\langle y(t), x(t)\rangle d t\right| \leq\|x\|_{\Phi}^{0}\|y\|_{\Psi}
\end{aligned}
$$

hold for any $x \in L_{\Phi}(X)$ and $y \in L_{\Psi}\left(X^{*}\right)$.

If $\Phi \in \Delta_{2}$, then $\left(L_{\Phi}(X)\right)^{*}=L_{\Psi}^{0}\left(X^{*}\right),\left(L_{\Phi}^{0}(X)\right)^{*}=L_{\Psi}\left(X^{*}\right)$ and the spaces $L_{\Phi}(X)$ and $L_{\Phi}^{0}(X)$ are reflexive if and only if $\Phi \in \Delta_{2} \cap \nabla_{2}$ (see [4] or [12]).

The Amemiya formula for the Orlicz norm

$$
\|x\|_{\Phi}^{0}=\inf _{k>0} \frac{1}{k}\left[1+\rho_{\Phi}(k x)\right]
$$

holds for every $x \in L_{\Phi}(X)$. Moreover, for every $x \in L_{\Phi}(X) \backslash\{0\}$ there exists $k>0$ such that

$$
\|x\|_{\Phi}^{0}=\frac{1}{k}\left[1+\rho_{\Phi}(k x)\right] .
$$

If there exists $k>0$ such that

$$
\int_{G} \Psi\left[\varphi\left(k\|x(t)\|_{X}\right)\right] d t=1,
$$

then

$$
\|x\|_{\Phi}^{0}=\int_{G}\|x(t)\|_{X} \varphi\left(k\|x(t)\|_{X}\right) d t=\frac{1}{k}\left\{1+\rho_{\Phi}(k x)\right\}
$$

(see [11]).

Now, we recall some geometric concepts in Banach spaces.

For any Banach space $X$ denote by $S(X)$ the unit sphere of $X$. The multi-valued mapping $\Lambda_{X}: X \backslash\{0\} \rightarrow S\left(X^{*}\right)$ defined by the formula

$$
\Lambda_{X}(x)=\left\{x^{*} \in S\left(X^{*}\right):\left\langle x^{*}, x\right\rangle=\|x\|_{X}\right\}
$$

for any $x \in X \backslash\{0\}$ is called the support mapping of $X$. The multi-valued mapping $F_{X}$ : $X \rightarrow X^{*}$ defined by the formula

$$
F_{X}(x)=\left\{x^{*} \in X^{*}:\left\langle x^{*}, x\right\rangle=\left\|x^{*}\right\|_{X}^{2}=\|x\|_{X}^{2}\right\}
$$

for any $x \in X$ is called the duality mapping of $X$. A relationship between the support mapping $\Lambda_{X}$ and the duality mapping $F_{X}$ can be expressed by the following formula:

$$
F_{X}(x)=\|x\|_{X} \Lambda_{X}(x) \quad \forall x \in X \backslash\{0\} \text { and } F_{X}(0)=0 .
$$

The properties of the duality mapping are closely related to the geometric properties of the space. The following results may be found in [3]: $F_{X}$ is homogeneous; $F_{X}$ is surjective iff $X$ is reflexive; $F_{X}$ is injective iff $X$ is strictly convex; $F_{X}$ is single-valued iff $X$ is smooth.

Now, we recall the concepts of the metric projection and the generalized projection. 
Let $C$ be a convex subset of a normed linear space $X$. The multi-valued mapping $\pi(C \mid \cdot): X \rightarrow C$ defined by the formula

$$
\pi(C \mid x)=\left\{x_{0} \in C:\left\|x-x_{0}\right\|_{X}=\inf _{z \in C}\|x-z\|_{X}\right\}
$$

for any $x \in X$ is called the metric projection onto $C$. If $\pi(C \mid \cdot)$ is single-valued, then it is called the metric projection operator or the best approximation operator and it is denoted by $\pi_{C}($ see $[13])$.

In the following we assume that $X$ is a reflexive, strictly convex and smooth Banach space. Consider the problem of the attainability of

$$
\inf _{y \in C}\left\{\|x\|_{X}^{2}-2\left\langle F_{X}(x), y\right\rangle+\|y\|_{X}^{2}\right\}
$$

We know that this problem has a unique solution (see [2]). The operator

$$
\Pi_{C} x:=\left\{y_{x} \in C: W\left(x, y_{x}\right)=\min _{y \in C} W(x, y)\right\}
$$

where $W(x, y)=\|x\|_{X}^{2}-2\left\langle F_{X} x, y\right\rangle+\|y\|_{X}^{2}$ for $x, y \in X$, is said to be the generalized projection of $x$ on $C$. Alber ([1]) obtained the following result:

THEOREM A. Let $X$ be a reflexive strictly convex smooth real Banach space, $K$ be a nonempty closed convex cone in $X$ (i.e. $\lambda K \subset K$ for all $\lambda \geq 0$ and $K+K=K$ ). Then for every $x \in X$ and $x^{*} \in X^{*}$ there exist $\omega \in K$ and $\chi \in K^{0}$, satisfying

$$
\begin{gathered}
x=F_{X}^{-1} \Pi_{K^{0}} F_{X}(x)+\omega \text { and }\left\langle\pi_{K^{0}} F_{X}(x), \omega\right\rangle=0, \\
x^{*}=F_{X} \Pi_{K} F_{X}^{-1}\left(x^{*}\right)+\chi \text { and }\left\langle\chi, F_{X}^{-1}\left(x^{*}\right)\right\rangle=0,
\end{gathered}
$$

where $K^{0}=\left\{x^{*} \in X^{*}:\left\langle x^{*}, x\right\rangle \leq 0 \quad \forall x \in K\right\}$ is the polar cone of $K$.

\section{A representation of the duality mapping}

Theorem 1. Let $\Phi \in \Delta_{2}, \varphi$ be continuous and $X$ be a smooth Banach space. Then the duality mapping $F_{L_{\Phi}^{0}(X)}$ of the Orlicz-Bochner space $L_{\Phi}^{0}(X)$ can be represented by the formula

$$
F_{L_{\Phi}^{0}(X)}(x)(t)=\|x\|_{\Phi}^{0} \Lambda_{X}(x(t)) \varphi\left[k\|x(t)\|_{X}\right]
$$

for $\mu$-a.e. $t \in G$ and for any $x \in L_{\Phi}^{0}(X)$, where $k$ satisfies

$$
\int_{G} \Psi\left[\varphi\left(k\|x(t)\|_{X}\right)\right] d t=1 .
$$

Proof. Let $Y=L_{\Phi}^{0}(X)$. Then we know that $Y^{*}=L_{\Psi}\left(X^{*}\right)$. Since $\Phi \in \Delta_{2}, \varphi$ is continuous and $X$ is smooth, by Th. 4 in [11], $Y=L_{\Phi}^{0}(X)$ is a smooth Banach space. Consequently, $F_{Y}: L_{\Phi}^{0}(X) \rightarrow L_{\Psi}\left(X^{*}\right)$ is a single-valued mapping and, by $(3), F_{Y}(x)=\|x\|_{\Phi}^{0} \Lambda_{Y}(x)$ for any $x \in L_{\Phi}^{0}(X) \backslash\{0\}$. 
By (1) and because of $\left\|\Lambda_{Y}(x)\right\|_{\Psi}=1$, there exists $k>0$ such that

$$
\begin{aligned}
\frac{1}{k}\left(1+\int_{G} \Phi\left(k\|x(t)\|_{X}\right) d t\right) & =\|x\|_{\Phi}^{0}=\int_{G}\left\langle\Lambda_{Y}(x)(t), x(t)\right\rangle d t \\
& \leq \frac{1}{k} \int_{G} k\|x(t)\|_{X}\left\|\Lambda_{Y}(x)(t)\right\|_{X^{*}} d t \\
& \leq \frac{1}{k}\left(\int_{G} \Phi\left(k\|x(t)\|_{X}\right) d t+\int_{G} \Psi\left(\left\|\Lambda_{Y}(x)(t)\right\|_{X^{*}}\right) d t\right) \\
& \leq \frac{1}{k}\left(\int_{G} \Phi\left(k\|x(t)\|_{X}\right) d t+1\right) .
\end{aligned}
$$

Hence

$$
\int_{G} \Psi\left(\left\|\Lambda_{Y}(x)(t)\right\|_{X^{*}}\right) d t=1
$$

and

$$
\int_{G}\left[\Phi\left(k\|x(t)\|_{X} \|\right)+\Psi\left(\left\|\Lambda_{Y}(x)(t)\right\|_{X^{*}}\right)-k\|x(t)\|_{X}\left\|\Lambda_{Y}(x)(t)\right\|_{X^{*}}\right] d t=0 .
$$

It follows from the Young inequality that

$$
\Phi\left(k\|x(t)\|_{X} \|\right)+\Psi\left(\left\|\Lambda_{Y}(x)(t)\right\|_{X^{*}}\right)=k\|x(t)\|_{X}\left\|\Lambda_{Y}(x)(t)\right\|_{X^{*}}
$$

for $\mu$-a.e. $t \in G$. The fact that $\varphi$ is continuous, and the condition for equality in the Young inequality yield that

$$
\left\|\Lambda_{Y}(x)(t)\right\|_{X^{*}}=\varphi\left(k\|x(t)\|_{X}\right)
$$

for $\mu$-a.e. $t \in G$. Therefore, we have

$$
\begin{aligned}
\int_{G}\left\langle\Lambda_{Y}(x)(t), k x(t)\right\rangle d t & =\int_{G} k\|x(t)\|_{X}\left\|\Lambda_{Y}(x)(t)\right\|_{X^{*}} d t \\
& =\int_{G} k\|x(t)\|_{X} \varphi\left(k\|x(t)\|_{X}\right) d t \\
& =\int_{G}\left\langle\varphi\left(k\|x(t)\|_{X}\right) \Lambda_{X}(x(t)), k x(t)\right\rangle d t .
\end{aligned}
$$

Since the map $x \mapsto \Lambda_{Y}(x)$ is single-valued, we obtain

$$
\Lambda_{Y}(x)(t)=\varphi\left(k\|x(t)\|_{X}\right) \Lambda_{X}(x(t))
$$

for $\mu$-a.e. $t \in G$. Combining (4) and (5), we get

$$
\int_{G} \Psi\left(\varphi\left(k\|x(t)\|_{X}\right)\right) d t=1
$$

and from (5) and the relationship between the duality mapping and the support mapping, we have

$$
F_{L_{\Phi}^{0}(X)}(x)(t)=\|x\|_{\Phi}^{0} \varphi\left[k\|x(t)\|_{X} \|\right] \Lambda_{X}(x(t))
$$

for $\mu$-a.e. $t \in G$.

Theorem 2. Let $\Phi \in \Delta_{2}, \varphi$ be continuous and $X$ be a smooth Banach space. Then the duality mapping $F_{L_{\Phi}(X)}$ of the Orlicz-Bochner space $L_{\Phi}(X)$ can be represented by the 
formula

$$
F_{L_{\Phi}(X)}(x)(t)=\frac{\|x\|_{\Phi}^{2}}{\int_{G}\|x(t)\|_{X} \varphi\left(\frac{\|x(t)\|_{X}}{\|x\|_{\Phi}}\right) d t} \varphi\left(\frac{\|x(t)\|_{X}}{\|x\|_{\Phi}}\right) \Lambda_{X}(x(t))
$$

for $\mu$-a.e. $t \in G$ and for every $x \in L_{\Phi}(X) \backslash\{0\}$.

Proof. Let $Y=L_{\Phi}(X)$. Then $Y^{*}=L_{\Psi}^{0}\left(X^{*}\right)$. Since $\Phi \in \Delta_{2}, \varphi$ is continuous, and $X$ is smooth, by Th. 3 in [11], the space $Y=L_{\Phi}(X)$ is a smooth Banach space. Consequently, the duality mapping $F_{Y}(\cdot)=\|\cdot\|_{\Phi} \Lambda_{Y}(\cdot)$ is single-valued.

Let $x \in L_{\Phi}(X) \backslash\{0\}$. Then $\Lambda_{Y}(x) \in S\left(L_{\Psi}^{0}\right)$ and

$$
\|x\|_{\Phi}=\int_{G}\left\langle\Lambda_{Y}(x)(t), x(t)\right\rangle d t .
$$

By (1), there is $k>0$ such that

$$
\begin{aligned}
\left\|\Lambda_{Y}(x)\right\|_{\Psi}^{0} & =1=\frac{1}{k}\left(1+\int_{G} \Psi\left(k\left\|\Lambda_{Y}(x)(t)\right\|_{X^{*}}\right) d t\right) \\
& =\int_{G}\left\langle\Lambda_{Y}(x)(t), \frac{x(t)}{\|x\|_{\Phi}}\right\rangle d t \\
& \leq \frac{1}{k} \int_{G} k\left\|\Lambda_{Y}(x)(t)\right\|_{X^{*}} \frac{\|x(t)\|_{X}}{\|x\|_{\Phi}} d t \\
& \leq \frac{1}{k}\left(\int_{G} \Psi\left(k\left\|\Lambda_{Y}(x)(t)\right\|_{X^{*}}\right) d t+\int_{G} \Phi\left(\frac{\|x(t)\|_{X}}{\|x\|_{\Phi}}\right) d t\right) \\
& \leq \frac{1}{k}\left(1+\int_{G} \Psi\left(k\left\|\Lambda_{Y}(x)(t)\right\|_{X^{*}}\right) d t\right) .
\end{aligned}
$$

It follows that

$$
k\left\|\Lambda_{Y}(x)(t)\right\|_{X^{*}} \frac{\|x(t)\|_{X}}{\|x\|_{\Phi}}=\Psi\left(k\left\|\Lambda_{Y}(x)(t)\right\|_{X^{*}}\right)+\Phi\left(\frac{\|x(t)\|_{X}}{\|x\|_{\Phi}}\right)
$$

for $\mu$-a.e. $t \in G$ and hence, by the continuity of $\varphi$ and by the condition for equality in the Young inequality, we obtain

$$
\left\|\Lambda_{Y}(x)(t)\right\|_{X^{*}}=\frac{1}{k} \varphi\left(\frac{\|x(t)\|_{X}}{\|x\|_{\Phi}}\right)
$$

for $\mu$-a.e. $t \in G$. By (6), we have

$$
\begin{aligned}
1 & =\int_{G}\left\langle\Lambda_{Y}(x)(t), \frac{x(t)}{\|x\|_{\Phi}}\right\rangle d t \\
& =\int_{G}\left\|\Lambda_{Y}(x)(t)\right\|_{X^{*}} \frac{\|x(t)\|_{X}}{\|x\|_{\Phi}} d t \\
& =\int_{G} \frac{1}{k} \varphi\left(\frac{\|x(t)\|_{X}}{\|x\|_{\Phi}}\right) \frac{\|x(t)\|_{X}}{\|x\|_{\Phi}} d t \\
& =\int_{G}\left\langle\frac{1}{k} \varphi\left(\frac{\|x(t)\|_{X}}{\|x\|_{\Phi}}\right) \Lambda_{X}(x(t)), \frac{x(t)}{\|x\|_{\Phi}}\right\rangle d t .
\end{aligned}
$$


Hence, it follows that

$$
\Lambda_{Y}(x)(t)=\frac{1}{k} \varphi\left(\frac{\|x(t)\|_{X}}{\|x\|_{\Phi}}\right) \Lambda_{X}(x(t))
$$

for $\mu$-a.e. $t \in G$. From (7), we see that

$$
k=\frac{1}{\|x\|_{\Phi}} \int_{G}\|x(t)\|_{X} \varphi\left(\frac{\|x(t)\|_{X}}{\|x\|_{\Phi}}\right) d t .
$$

Therefore, we obtain

$$
F_{L_{\Phi}}(x)(t)=\frac{\|x\|_{\Phi}^{2}}{\int_{G}\|x(t)\|_{X} \varphi\left(\frac{\|x(t)\|_{X}}{\|x\|_{\Phi}}\right) d t} \varphi\left(\frac{\|x(t)\|_{X}}{\|x\|_{\Phi}}\right) \Lambda_{X}(x(t))
$$

for $\mu$-a.e. $t \in G$.

\section{A generalized projection decomposition}

THEOREM 3. Let $\Phi \in \Delta_{2} \cap \nabla_{2}, \varphi$ and $\psi$ be continuous, $X$ be a reflexive strictly convex smooth Banach space, $K$ be a nonempty closed convex cone in the Orlicz-Bochner space $L_{\Phi}(X), K^{0}=\left\{y \in L_{\Psi}^{0}\left(X^{*}\right): \int_{G}\langle y(t), x(t)\rangle d t \leq 0 \forall x \in K\right\}$. Then for any $x \in L_{\Phi}(X) \backslash K$, we have the unique decomposition

$$
x(t)=\pi_{K}(x)(t)+\|y\|_{\Psi}^{0} \Lambda_{X^{*}}(y(t)) \psi\left(k\|y(t)\|_{X^{*}}\right)
$$

for $\mu$-a.e. $t \in G$, where $k>0$ and $y \in L_{\Psi}^{0}\left(X^{*}\right)$ satisfy the conditions

$$
\int_{G} \Phi\left[\psi\left(k\|y(t)\|_{X^{*}}\right)\right] d t=1
$$

and

$$
y(t)=\Pi_{K^{0}}\left(\frac{\|x\|_{\Phi}^{2}}{\int_{G}\|x(t)\|_{X} \varphi\left(\frac{\|x(t)\|_{X}}{\|x\|_{\Phi}}\right) d t} \varphi\left(\frac{\|x(.)\|_{X}}{\|x\|_{\Phi}}\right) \Lambda_{X}(x(.))\right)(t)
$$

for $\mu$-a.e. $t \in G$, where $\pi_{K}$ is the metric projection operator from $L_{\Phi}$ onto $K$ and $\Pi_{K^{0}}$ is the generalized projection operator from $L_{\Psi}^{0}\left(X^{*}\right)$ onto $K^{0}$.

Proof. Let $Y=L_{\Phi}(X)$. Then $Y^{*}=L_{\Psi}^{0}\left(X^{*}\right)$ and both $L_{\Phi}(X)$ and $L_{\Psi}^{0}\left(X^{*}\right)$ are reflexive, strictly convex and smooth spaces. For any $x \in Y \backslash K$, by Theorem A, there exists a function $\omega \in K$ such that

$$
x=\omega+F_{Y}^{-1} \Pi_{K^{0}} F_{Y}(x) \text { and }\left\langle\Pi_{K^{0}} F_{Y}(x), \omega\right\rangle=0 .
$$

Hence, we have

$$
\begin{gathered}
F_{Y}(x-\omega)=\Pi_{K^{0}} F_{Y}(x) \in K^{0}, \\
\left\langle F_{Y}(x-\omega), \omega\right\rangle=0 \text { and }\left\langle F_{Y}(x-\omega), w\right\rangle \leq 0
\end{gathered}
$$

for any $w \in K$. By Theorem 2, we obtain

$$
\int_{G}\left\langle\varphi\left(\frac{\|x(t)-\omega(t)\|_{X}}{\|x-\omega\|_{\Phi}}\right) \Lambda_{X}(x(t)-\omega(t)), \omega(t)-w(t)\right\rangle d t \geq 0
$$

for any $w \in K$. It follows from Theorem 6 in [11] that

$$
\omega=\pi_{K}(x) \text {. }
$$


By Theorem 2, we get

$$
F_{Y}(x)(t)=\frac{\|x\|_{\Phi}^{2}}{\int_{G}\|x(t)\|_{X} \varphi\left(\frac{\|x(t)\|_{X}}{\|x\|_{\Phi}}\right) d t} \varphi\left(\frac{\|x(t)\|_{X}}{\|x\|_{\Phi}}\right) \Lambda_{X}(x(t))
$$

and

$$
y(t)=\Pi_{K^{0}} F_{Y}(x)(t)=\Pi_{K^{0}}\left(\frac{\|x\|_{\Phi}^{2}}{\int_{G}\|x(t)\|_{X} \varphi\left(\frac{\|x(t)\|_{X}}{\|x\|_{\Phi}}\right) d t} \varphi\left(\frac{\|x(\cdot)\|_{X}}{\|x\|_{\Phi}}\right) \Lambda_{X}(x(\cdot))\right)(t)
$$

for $\mu$-a.e. $t \in G$. The fact that $Y$ and $Y^{*}$ are reflexive, strictly convex and smooth Banach spaces implies that $F_{Y}^{-1}=F_{Y^{*}}$ and hence, by Theorem 1, we have

$$
F_{Y}^{-1} \Pi_{K^{0}} F_{Y}(x)(t)=F_{Y^{*}}(y)(t)=\|y\|_{\Psi}^{o} \Lambda_{X^{*}}(y(t)) \psi\left(k\|y(t)\|_{X^{*}}\right)
$$

for $\mu$-a.e. $t \in G$ and

$$
\int_{G} \Phi\left[\psi\left(k\|y(t)\|_{X^{*}}\right)\right] d t=1
$$

Combining (8), (9) and (10), we finish the proof.

Corollary 1 (Moreau decomposition theorem). Let $X$ be a Hilbert space, $K \subset L^{2}(X)$ be a closed convex cone, $K^{0} \subset L^{2}(X)$ be its polar cone. Then for every $x \in L^{2}(X) \backslash K$, there is a unique decomposition

$$
x=\pi_{K}(x)+\pi_{K^{0}}(x),
$$

where $\pi_{K}$ and $\pi_{K^{0}}$ are the metric projection operators.

Proof. Let $\Phi(u)=|u|^{2} / 2$. Then $\Psi(v)=|v|^{2} / 2$. Since $X$ is a Hilbert space, $Y=$ $L_{\Phi}(X)=L^{2}(X)$ and $Y^{*}=L_{\Psi}^{0}\left(X^{*}\right)=L^{2}(X)$. Moreover, for any $x \in L_{\Phi}(X)$ and for any $y \in L_{\Psi}^{0}\left(X^{*}\right)$, we have $\|x\|_{\Phi}=\|x\|_{2} / \sqrt{2}$ and $\|y\|_{\Psi}^{0}=\sqrt{2}\|y\|_{2}$. Consequently, for any $x \in L_{\Phi}(X) \backslash K$, we have

$$
\begin{aligned}
F_{Y}(x)(t) & =\frac{\|x\|_{\Phi}^{2}}{\int_{G}\|x(t)\|_{X} \varphi\left(\frac{\|x(t)\|_{X}}{\|x\|_{\Phi}}\right) d t} \varphi\left(\frac{\|x(t)\|_{X}}{\|x\|_{\Phi}}\right) \Lambda_{X}(x(t)) \\
& =\frac{\|x\|_{2}^{2}}{\int_{G}\|x(t)\|_{X}^{2} d t} \cdot \frac{\sqrt{2}\|x\|_{2}}{2 \sqrt{2}} \cdot \frac{\|x(t)\|_{X}}{\|x\|_{2}} \Lambda_{X}(x(t))=\frac{1}{2}\|x(t)\|_{X} \Lambda_{X}(x(t))=\frac{1}{2} x(t)
\end{aligned}
$$

for $\mu$-a.e. $t \in G$. Since in any Hilbert space, the generalized projection operator $\Pi_{K^{0}}$ coincides with the metric projection operator $\pi_{K^{0}}$,

$$
y(t)=\Pi_{K^{0}} F_{Y}(x)(t)=\frac{1}{2} \pi_{K^{0}}(x)(t)
$$

for $\mu$-a.e. $t \in G$. On the other hand, we also have

$$
\|y\|_{\Psi}^{0} \psi\left[k\|y(t)\|_{X^{*}}\right] \Lambda_{X^{*}}(y(t))=\sqrt{2}\|y\|_{2} k\|y(t)\|_{X} \Lambda_{X}(y(t))=\sqrt{2}\|y\|_{2} k y(t) .
$$

From the condition

$$
1=\int_{G} \Phi\left[\psi\left(k\|y(t)\|_{X^{*}}\right)\right] d t=\frac{k^{2}}{2} \int_{G}\|y(t)\|_{X}^{2} d t
$$

we get that $k\|y\|_{2}=\sqrt{2}$, and so

$$
\|y\|_{\Psi}^{0} \psi\left[k\|y(t)\|_{X^{*}}\right] \Lambda_{X^{*}}(y(t))=2 y(t)=\pi_{K^{0}}(x)(t) .
$$


Hence and from Theorem 3, we get

$$
x(t)=\pi_{K}(x)(t)+\pi_{K^{0}}(x)(t)
$$

for $\mu$-a.e. $t \in G$.

By Corollary 1, we obtain immediately the following

Corollary 2 (Riesz orthogonal decomposition theorem). Let $X$ be a Hilbert space, $L \subset L^{2}(X)$ be a closed linear subspace, $L^{\perp} \subset L^{2}(X)$ be its orthogonal complement. Then for every $x \in L^{2}(X) \backslash L$ there is a unique decomposition

$$
x=P_{L}(x)+P_{L^{\perp}}(x),
$$

where $P_{L}$ and $P_{L^{\perp}}$ are the orthogonal projection operators.

Now we will give an application of Theorem 3. Namely, we have the representation of the metric projection operator onto a closed hyperplane in Orlicz-Bochner spaces.

TheOREM 4. Let $\Phi \in \Delta_{2} \cap \nabla_{2}, \varphi$ and $\psi$ be continuous, $X$ be a reflexive, strictly convex and smooth Banach space. Let $L=\left\{x \in L_{\Phi}(X): \int_{G}\left\langle x_{0}^{*}(t), x(t)\right\rangle d t=0\right\}$ be a closed hyperplane in $L_{\Phi}(X)$, where $x_{0}^{*} \in L_{\Psi}^{0}\left(X^{*}\right) \backslash\{0\}$. Then for every $x \in L_{\Phi}(X) \backslash L$, we have

$$
\pi_{L}(x)(t)=x(t)-\frac{\int_{G}\left\langle x_{0}^{*}(t), x(t)\right\rangle d t}{\|x\|_{\Psi}^{0}} \psi\left[k\left\|x_{0}^{*}(t)\right\|_{X^{*}}\right] \Lambda_{X^{*}}\left(x_{0}^{*}(t)\right),
$$

for $\mu$-a.e. $t \in G$, where $\Lambda_{X^{*}}$ is the support mapping of $X^{*}$.

Proof. By the assumptions, we have that the Orlicz-Bochner space $Y=L_{\Phi}(X)$ is a reflexive, strictly convex and smooth Banach space. For the closed hyperplane $L$, we know that

$$
L^{0}=L^{\perp}=\left\{\lambda x_{0}^{*}: \lambda \in R\right\} \subset L_{\Psi}^{o}\left(X^{*}\right)=Y^{*} .
$$

For any $x \in L_{\Phi}(X) \backslash L$, by Theorem 3, we have

$$
\pi_{L}(x)(t)=x(t)-F_{Y^{*}}(y)(t),
$$

where $y=\lambda x_{0}^{*} \in L^{\perp}$ for some $\lambda \neq 0$. Note that the duality mapping $F_{Y^{*}}$ is homogeneous, so we obtain

$$
\pi_{L}(x)(t)=x(t)-\lambda F_{Y}^{-1}\left(x_{0}^{*}\right)(t) .
$$

Taking the value of the functional $x_{0}^{*}(t)$ at the elements from both sides of (11) for every $t \in G$ and then integrating them over $G$ with respect to $t \in G$, we get

$$
0=\int_{G}\left\langle x_{0}^{*}(t), x(t)\right\rangle d t-\lambda \int_{G}\left\langle x_{0}^{*}(t), F_{Y}^{-1}\left(x_{0}^{*}\right)(t)\right\rangle d t=\int_{G}\left\langle x_{0}^{*}(t), x(t)\right\rangle d t-\lambda\left(\left\|x_{0}^{*}\right\|_{\Psi}^{0}\right)^{2} .
$$

Hence, it follows that

$$
\lambda=\frac{\int_{G}\left\langle x_{0}^{*}(t), x(t)\right\rangle d t}{\left(\left\|x_{0}^{*}\right\|_{\Psi}^{0}\right)^{2}}
$$

On the other hand, by Theorem 1 , we also have

$$
F_{Y}^{-1}\left(x_{0}^{*}\right)(t)=F_{Y^{*}}\left(x_{0}^{*}\right)(t)=\left\|x_{0}^{*}\right\|_{\Psi}^{0} \psi\left[k\left\|x_{0}^{*}(t)\right\|_{X^{*}}\right] \Lambda_{X^{*}}\left(x_{0}^{*}(t)\right)
$$

Combining (11), (12) and (13), we complete the proof. 


\section{References}

[1] Ya. I. Alber, Generalized projections decompositions and the Pythagorean-type theorem in Banach spaces, Appl. Math. Lett. 11 (1998), 115-121.

[2] Ya. I. Alber, Metric and generalized projection operators in Banach spaces: properties and applications, in: Theory and Applications of Nonlinear Operators of Monotone and Accretive Type, A. Kartsatos (ed.), Marcel Dekker, New York, 1996, 15-50.

[3] V. Barbu and T. Precupanu, Convexity and Optimization in Banach Spaces, Ed. Aca. Rep. Soc. Romania, Bucureşti, 1978.

[4] S. Chen, Geometry of Orlicz spaces, Dissertationes Mathematicae 356 (1996).

[5] J. Diestel, Geometry of Banach Spaces-Selected Topics, Lecture Notes in Math. 485, Springer, New York, 1975.

[6] H. Hudzik, Strict convexity of Musielak-Orlicz space with Luxemburg norm, Bull. Polish Acad. Sci. Math. 29 (1981), 338-367.

[7] A. Kozek, Orlicz spaces of functions with values in Banach spaces, Comment. Math. 19 (1976), 259-288.

[8] M. A. Krasnosel'skiı̌ and Ya. B. Rutickiǔ, Convex Functions and Orlicz Spaces, Noordhof, Groningen, 1961.

[9] J. J. Moreau, Décomposition orthogonale d'un espace hilbertien selon deux cônes mutuellement polaires, C. R. Acad. Sci. Paris 255 (1962), 238-240.

[10] J. Musielak, Orlicz Spaces and Modular Spaces, Lecture Notes in Math. 1034, Springer, 1983.

[11] R. Płuciennik and Y. W. Wang, Metric projections and best approximants in BochnerOrlicz spaces, Revista Matemática Complutense 7 (1994), 57-77.

[12] M. M. Rao and Z. D. Ren, Theory of Orlicz Spaces, Marcel Dekker, New York, 1991.

[13] I. Singer, The Theory of Best Approximation and Functional Analysis, Springer-Verlag, New York, 1970.

[14] W. Song and Z. J. Cao, The generalized projection decomposition theorem in Banach spaces and its applications, J. Approx. Theory 129 (2004), 167-181.

[15] Y. W. Wang and Z. W. Li, The Moore-Penrose generalized inverse in Banach spaces and ill-posed problem, J. Sys. Sci. 15 (1995), 175-185.

[16] Y. W. Wang and H. Wang, Generalized orthogonal decomposition theorem in Banach spaces and generalized orthogonally complemented subspace, Acta Math. Sinica 44 (2001), 1045-1050.

[17] C. X. Wu, T. F. Wang, S. T. Chen and Y. W. Wang, Geometry of Orlicz Spaces, Harbin (1986) (in Chinese). 